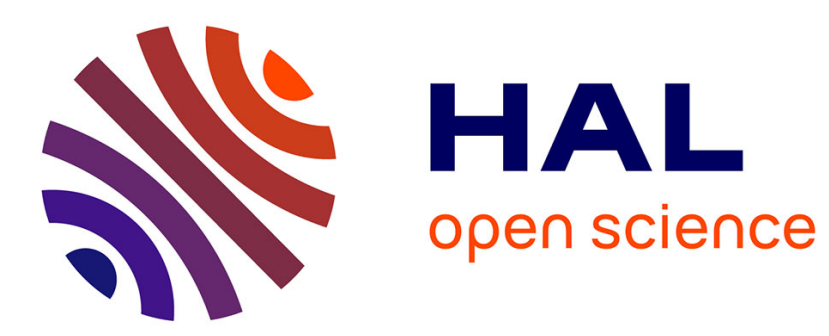

\title{
Euclidean Configuration Space Renormalization, Residues and Dilation Anomaly1
}

Nikolay M. Nikolov, Raymond Stora, Ivan Todorov

\section{To cite this version:}

Nikolay M. Nikolov, Raymond Stora, Ivan Todorov. Euclidean Configuration Space Renormalization, Residues and Dilation Anomaly1. 2012. hal-00703709

\section{HAL Id: hal-00703709 \\ https://hal.science/hal-00703709}

Preprint submitted on 4 Jun 2012

HAL is a multi-disciplinary open access archive for the deposit and dissemination of scientific research documents, whether they are published or not. The documents may come from teaching and research institutions in France or abroad, or from public or private research centers.
L'archive ouverte pluridisciplinaire HAL, est destinée au dépôt et à la diffusion de documents scientifiques de niveau recherche, publiés ou non, émanant des établissements d'enseignement et de recherche français ou étrangers, des laboratoires publics ou privés. 
CERN-TH-PH/2012-076

LAPTH-Conf-016/12

\title{
Euclidean Configuration Space Renormalization, Residues and Dilation Anomaly ${ }^{1}$
}

\author{
Nikolay M. Nikolov \\ Institute for Nuclear Research and Nuclear Energy \\ Tsarigradsko Chaussee 72, BG-1784 Sofia, Bulgaria \\ mitov@inrne.bas.bg \\ Raymond Stora \\ Laboratoire d'Annecy-le-Vieux de Physique Théorique (LAPTH) \\ F-74941 Annecy-le-Vieux Cedex, France \\ and
}

Theory Division, Department of Physics, CERN, CH-1211 Geneva 23, Switzerland

Ivan Todorov

Institut des Hautes Études Scientifiques, F-91440 Bures-sur-Yvette, France

and

Theory Division, Department of Physics, CERN, CH-1211 Geneva 23, Switzerland permanent address:

Institute for Nuclear Research and Nuclear Energy

Tsarigradsko Chaussee 72, BG-1784 Sofia, Bulgaria

todorov@inrne.bas.bg

\begin{abstract}
Configuration $(x$-)space renormalization of euclidean Feynman amplitudes in a massless quantum field theory is reduced to the study of local extensions of associate homogeneous distributions. Primitively divergent graphs are renormalized, in particular, by subtracting the residue of an analytically regularized expression. Examples are given of computing residues that involve zeta values. The renormalized Green functions are again associate homogeneous distributions of the same degree that transform under indecomposable representations of the dilation group.
\end{abstract}

\footnotetext{
${ }^{1}$ Invited lecture by I. Todorov at the 9. International Workshop Lie Theory and Its Applications in Physics (LT-9), Varna, Bulgaria, June 2011, and at the International Workshop "Supersymmetries and Quantum Symmetries" (SQS'2011), Dubna, Russia, July 2011.
} 


\section{Contents}

1 Introduction $\quad 2$

2 General requirements. Reduction to a one-dimensional problem

3 Residues and renormalization of primitively divergent graphs 10

4 Dilation anomaly. Examples of graphs with subdivergences 14

5 Concluding remarks $\quad 17$

$\begin{array}{lll}\text { Appendix A Radial associate homogeneous distributions } & 19\end{array}$ 


\section{Introduction}

Fourier transform is a prime example of the now fashionable notion of duality. It maps a problem of integrating large momenta into one of studying the short distance behaviour of correlation functions. Divergences were first discovered and renormalization theory was developed for momentum space integration. E.C.G. Stueckelberg and A. Petermann [SP], followed by N.N. Bogolubov, a mathematician who set himself to master quantum field theory (QFT), realized that (perturbative) renormalization can be formulated as a problem of extending products of distributions, originally defined for non-coinciding arguments ${ }^{2}$ and that such extensions are naturally restricted by locality or micro-causality (a concept introduced in QFT by Stueckelberg [Stu] and further developed by Bogolubov and collaborators - for a review and references see [BS]). The idea was taken up and implemented systematically by H. Epstein and V. Glaser [EG] [EGS](see also parallel work by O. Steinmann [St]; for later contibutions and surveys see [S82/93] [S06]). It is conceptually clear and represents a crucial step in turning QFT renormalization into a mathematically respectable theory. By the late 1990's when the problem of developing perturbative QFT and operator product expansions on a curved background became the order of the day, it was realized that it is just the $x$-space approach that offers a way to its solution [BF, BFV, DF, H07, H08, HW, HW08]. It is therefore not surprising that this approach attracts more attention now than half a century ago when it was originally conceived - see e.g. [G-B, G-BL, FG-B, EGP, FHS, K, N, K10, B]. Papers like $[\mathrm{BBK}]$ reflect, surely, later developments in both renormalization theory (Kreimer's Hopf algebra structure - see e.g. [Kr] - and Connes-Kreimer's reduction to the Riemann-Hilbert's problem [CK]) and the mathematical study of singularities in configuration space [FM, DP]. Recent work on Feynman graphs and motives [BEK, BK] also generated a configuration space development $[\mathrm{Ni}, \mathrm{N}, \mathrm{CM}]$.

A starting point in our work was the observation (cf. [BF], [HW], [G-B], $[\mathrm{DF}])$ that Hörmander's treatment of the extension of homogeneous distributions (Sect. 3.2 of $[\mathrm{H}]$ ) is tailor-made for treating the ultraviolet (UV) renormalization problem, that is particularly transparent in a massless QFT. In order to explain the main ideas stripped of technicalities, we begin with the study of dilation invariant euclidean Green's functions (the only case considered in [BBK]). Furthermore, we concentrate on the UV problem excluding integration in configuration space by considering all vertices as external.The validity of the results in the physically better motivated Minkowski space framework is established in [NST]. It is, on the other hand, known that the leading UV singularities in a massive QFT are given by the corresponding massless limit. The full study of the renormalization problem in the massive case requires, however, additional steps and is relegated to future work.

\footnotetext{
${ }^{2}$ Whereas $x$-space renormalization was straightened out in all generality [BP] [Step] [Hep], it took some more time to settle the $p$-space problem [Z] [Zi] [L] [LZ], resulting in what is now termed the BPHZ theory.
} 
We begin with a framework that differs from standard QFT (cf. [Ni]). We separate the renormalization program from concrete (massless) QFT models and state it as a mathematical problem of extension of a class of homogeneous distributions. In Sect. 2 we formulate general axiomatic conditions for our construction, such that when combined with a given Lagrangian model it reproduces the result of Epstein-Glaser for the renormalized time ordered products (see $[\mathrm{N}]$ ). To this end we introduce a universal algebra of rational translation invariant functions in $\mathbb{R}^{D n}$, where $n$ runs in $\mathbb{N}$ while $D$, the space-time dimension, is fixed ( $D=4$ being the case of chief interest). We assume that this algebra is generated by 2-point functions of the type

$$
\begin{array}{r}
G_{i j}\left(x_{i j}\right)=\frac{P_{i j}\left(x_{i j}\right)}{\rho_{i j}^{\mu_{i j}}}, \quad x_{i j}=x_{i}-x_{j}, \quad \mu_{i j} \in \mathbb{N}, \\
\rho_{i j}=\left|x_{i j}\right|=\left(x_{i j}^{2}\right)^{1 / 2}, x^{2}=\sum_{\alpha=1}^{D}\left(x^{\alpha}\right)^{2}
\end{array}
$$

(for a Minkowski space signature $\left.\left.\rho^{2}=\mathbf{x}^{2}-\left(x^{0}-i 0\right)^{2}\right), \mathbf{x}^{2}=\sum_{i=1}^{D-1}\left(x^{i}\right)^{2}\right)$; here $P_{i j}$ are homogeneous polynomials in the components of the $D$-vector $x_{i j}$. (For free massless fields in an odd dimensional spacetime the exponents $\mu_{i j}$ are odd $^{3}$. For an even D one can assume that all $\mu_{i j}$ are even integers so that $G_{i j}$ are rational fumctions.) We note that the renormalization of any massless QFT can be reduced to the extension of (a subspace of) rational functions $G=$ $\prod_{i<j} G_{i j}\left(x_{i j}\right)$ of this algebra to distributions on $\mathbb{R}^{D(n-1)}$. The correspondence between the rational functions and such distributions is called a renormalization map. Each expression

$$
G_{\Gamma}=\prod_{(i j) \in \Gamma} G_{i j}\left(x_{i j}\right)
$$

can be represented by a decorated graph $\Gamma$ of $n$ vertices and of lines connecting pairs of different vertices $(i, j)$ whenever there is a (non-constant) factor $G_{i j}$ in the product (1.2). Each $G_{i j}=G_{i j}\left(x_{i j}\right)$ appears at most once in this expression, so that there are no multiple lines in the graph $\Gamma$. The presence of different powers $\mu$ and different polynomials $P$ indicates the fact that we give room for composite fields in our theory such as normal products of derivatives of the basic fields. (Matrix valued vertices that enter the Feynman rules can be accounted for by admitting linear combinations of expressions of type (1.2).) A disconnected graph $\Gamma$ corresponds to the (tensor) product of the distributions associated to its connected components. We shall restrict our attention to connected graphs.

We remark that a quantum field theorist may wish to replace the polynomial in $\mathrm{x}$ in (1.1) by a polynomial of derivatives acting on the scalar field propagator. The difference is not accidental: we shall impose the requirement, convenient for the subsequent analysis, that the renormalization map commutes with multiplication by polynomials in $x_{i j}$. On the other hand, derivatives typically yield

\footnotetext{
${ }^{3}$ In view of recent interest in 3D CFT [GPY] [MZ] we explicitely include here odd D.
} 
anomalies independently of the above requirement (see $[\mathrm{N}]$, Sect. 8). Using the renormalization map we achieve the basic property of the time-ordered product: causality. Other constraints compatible with causality and power counting may be imposed - including a description of possible associated anomalies - by adjustment of additional finite renormalizations. An example of such a phenomenon, concerned with the behaviour of renormalized Feynman amplitudes under dilations, is considered in Sect. 4.

Thus, to any graph $\Gamma$ in a given massless QFT there corresponds a bare Feynman amplitude $G_{\Gamma}$. It is a homogeneous rational function of degree $-d_{\Gamma}$ which depends on n-1 $D$-vector differences. We shall denote the arguments of $G_{\Gamma}$ by $\vec{x}$, for short, and will introduce a uniform ordering $x^{1}, \ldots, x^{N}$ of their components, where $N=D(n-1)$ (for a connected graph). Then, the homogeneity of $G_{\Gamma}$ is expressed as

$$
G_{\Gamma}(\lambda \vec{x})=\lambda^{-d_{\Gamma}} G_{\Gamma}(\vec{x}) .
$$

We shall call the difference $\kappa:=d_{\Gamma}-N$ the index of divergence. It coincides with (minus) the degree of homogeneity of the density form

$$
G_{\Gamma}(\vec{x}) d x^{1} \wedge d x^{2} \wedge \ldots \wedge d x^{N} \equiv G_{\Gamma}(\vec{x}) \text { Vol. . }
$$

(Whenever the orientation is not relevant we shall skip the wedge product sign. The use of densities rather than functions streamlines changes of variables and partial integration.) We say that $G_{\Gamma}$ is superficially divergent if $\kappa \geq 0 ; G_{\Gamma}$ is called divergent if it is not locally integrable. The following easy to prove statement justifies the above terminology.

Proposition 1.1. If the indices of divergence of a connected graph $\Gamma$ and of all its connected subgraphs are negative then $G_{\Gamma}$ is locally integrable and admits, as a consequence, a unique continuation as a distribution on $\mathbb{R}^{D(n-1)}$.

The power counting index of divergence of standard renormalization theory is thus replaced by the degree of homogeneity of bare Green functions for a (classically dilation invariant) massless QFT.

Abusing the terminology we shall also speak of (superficially) divergent graphs. Each function $G_{\Gamma}$ defines a tempered distribution (in the sense of Schwartz $[\mathrm{Sc}]$ ) on test functions $f$ with support

$$
\operatorname{supp} f \subset \mathbb{R}^{D(n-1)} \backslash \Delta_{2}, \quad \Delta_{2}=\left\{\vec{x} ; \exists(i, j) i<j, \text { s.t. } x_{i j}=0\right\} .
$$

One can, similarly, introduce the partial diagonals $\Delta_{k}$ involving $k$-tuples of coinciding points; we have $\Delta_{n}:=\left\{\vec{x} ; x_{1}=\ldots=x_{n}\right\} \subset \Delta_{n-1} \subset \ldots \subset \Delta_{2}$. We shall be mostly using the small or full diagonal $\Delta_{n}$ in what follows. The problem of renormalization consists in extending all distributions $G_{\Gamma}$ to $\mathcal{S}\left(\mathbb{R}^{D(n-1)}\right)$ in such a way that a certain recursion relation, which reflects the causality condition, is satisfied. This condition is known as causal factorization. We give the precise formulation of its euclidean version in Sect. 2 that follows from the more involved but physically motivated Minkowski space requirement (see [NST]). 
We use an $x$-space counterpart of Speer's analytic renormalization in [Sp] to define the notion of residue $e^{4}$ of $G_{\Gamma}$ adapted, in particular, to primitively divergent graphs. It is based on the observation that if $r=r\left(x_{i j}\right)$ is a norm in the (euclidean) space of coordinate differences and $G(\vec{x})$ is primitively divergent of index $\kappa$ then the analytically regularized Feynman amplitude

$$
r^{\kappa+\epsilon} G(\vec{x})(\epsilon>0)
$$

is locally integrable. It will be proven in Sect. 2 and Appendix A that Eq. (1.6) defines a distribution valued meromorphic function in $\epsilon$ which only has simple poles for non-positive integer values of $\epsilon$. This will allow us to define the renormalized Feynman distribution $G^{R}$ of a primitively divergent graph by just subtracting the pole term for $\epsilon=0$. The result will be enforced by one of our main requirements (see (MC2) of Sect. 2, below), namely that $G^{R}$ is associate homogeneous of the same degree as $G$ (its behaviour for small $r$ only differing from $G$ by $\log$ terms). More precisely, we say that $G$ is an associate homogeneous distribution of degree $d$ and order $k$ if it obeys the (infinitesimal) indecomposable dilation law

$$
(E+d)^{k+1} G(\vec{x})=0 \quad \text { where } \quad E=\sum_{i=1}^{n} x_{i} \frac{\partial}{\partial x_{i}}\left(x \frac{\partial}{\partial x}=\sum_{\alpha=1}^{D} x^{\alpha} \frac{\partial}{\partial x^{\alpha}}\right),
$$

- i.e., if it is an associate eigenvector of the Euler operator $E$ - see [GS].

The study of divergent graphs with subdivergences is outlined in Sect. 4, where a global characterization of associate homogeneous distributions is also given. It is remarkable that in all cases renormalization is reduced to a 1dimensional extension problem for associate homogeneous distributions. A construction that provides the solution to this problem is outlined in Appendix A.

One objective of our work is to demonstrate in a systematic fashion that $x$-space calculations are not only more transparent conceptually but also practical (especially in the euclidean massless case - something noticed long ago by Chetyrkin et al. $[\mathrm{CKT}]$ (see also [KTV]) but only rarely appreciated afterwards - cf. [G-B]). To this end we consider (in Sects. 3, 4) a number of examples (of 1-, 2- and 3-loop graphs) displaying the basic simplicity of the argument. A primitively divergent $n$-loop graph whose residue involves $\zeta(2 n-3)$ is displayed as Example 3.2.

\footnotetext{
${ }^{4} \mathrm{~A}$ notion of residue of a Feynman graph has been introduced in the momentum space approach in terms of the graph polynomial [BEK, BK]. It would be interesting to establish the precise relationship between that notion and ours. The notion of Poincaré residue considered in $[\mathrm{CM}]$, on the other hand, works in a straightforward manner for simple poles in $x$-space, a rather unnatural restriction for ultraviolet divergences.
} 


\section{General requirements. Reduction to a one- dimensional problem}

We shall define ultraviolet (i.e. short distance) renormalization by induction with respect to the number of vertices. Assume that all contributions of diagrams with less than $n$ points are renormalized. If then $\Gamma$ is an arbitrary connected $n$-point graph its renormalized contribution should satisfy the following inductive factorization requirement.

Let the index set $I(n)=\{1, \ldots, n\}$ of $\Gamma$ be split into any two non-empty non-intersecting subsets

$$
I(n)=I_{1} \cup \dot{\cup} I_{2}\left(I_{1} \neq \emptyset, I_{2} \neq \emptyset, I_{1} \cap I_{2}=\emptyset\right) .
$$

Let $\mathcal{U}_{I_{1}, I_{2}}$ be the open subset of $\mathbb{R}^{D n} \equiv\left(\mathbb{R}^{D}\right)^{\times n}$ such that $\left(x_{1}, \ldots, x_{n}\right) \notin \mathcal{U}_{I_{1}, I_{2}}$ whenever there is a pair $(i, j)$ such that $i \in I_{1}, j \in I_{2}$. Let further $G_{1}^{R}$ and $G_{2}^{R}$ be the contributions of the subgraphs of $\Gamma$ with vertices in $I_{1}$ and $I_{2}$, respectively. For each such splitting our distribution $G_{\Gamma}^{R}$, defined on all partial diagonals, exhibits the euclidean factorization property $(-$ see $[\mathrm{Ni}])$ :

$$
G_{\Gamma}^{R}=G_{1}^{R}\left(\prod_{\substack{i \in I_{1} \\ j \in I_{2}}} G_{i j}\right) G_{2}^{R} \quad \text { on } \quad \mathcal{U}_{I_{1}, I_{2}},
$$

where $G_{i j}$ are factors (of type (1.1)) in the rational function $G_{\Gamma}$ and are understood as multipliers on $\mathcal{U}_{I_{1}, I_{2}}$. This property is inspired by the Minkowski space causal factorization of Epstein-Glaser [EG] considered in [NST].

We shall add to this basic physical requirement a few more mathematical conventions (MC) which will substantially restrict the notion of renormalization used in this paper.

(MC1) The renormalization commutes with permutation of indices (which may stand for both position variables and discrete quantum numbers).

(MC2) Renormalization maps rational homogeneous functions onto associate homogeneous distributions of the same degree of homogeneity; it extends associate homogeneous distributions defined off the small diagonal to associate homogeneous distributions of the same degree (but possibly of higher order) defined everywhere on $\mathbb{R}^{N}$.

(MC3) The renormalization map commutes with multiplication by (homogeneous) polynomials. If we extend the class of our distributions allowing multiplication with smooth functions of no more than polynomial growth (in the domain of definition of the corresponding functionals), then this requirement will imply commutativity of the renormalization map with such multipliers.

(MC4) In a euclidean invariant theory the renormalization map commutes with euclidean transformation in $\mathbb{R}^{D}$.

The induction is based on the following euclidean diagonal lemma. 
Proposition 2.1. The complement $C\left(\Delta_{n}\right)$ of the small diagonal is the union of all $\mathcal{U}_{I_{1}, I_{2}}$ for all pairs of disjoint $I_{1}, I_{2}$ with $I_{1} \cup I_{2}=\{1, \ldots, n\}$, i.e.,

$$
C\left(\Delta_{n}\right)=\bigcup_{I_{1} \cup I_{2}=\{1, \ldots, n\}} \mathcal{U}_{I_{1}, I_{2}}
$$

Proof. Let $\left(x_{1}, \ldots, x_{n}\right) \in C\left(\Delta_{n}\right)$. Then there are at least two different points $x_{i_{1}} \neq x_{j_{1}}$. We define $I_{1}$ as the set of all indices $i$ of $I=I(n)$ for which $x_{i} \neq x_{j_{1}}$ and $I_{2}:=I \backslash I_{1}$. Hence, $C\left(\Delta_{n}\right)$ is included in the union of all such pairs. Each $\mathcal{U}_{I_{1}, I_{2}}$, on the other hand, is defined to belong to $C\left(\Delta_{n}\right)$. This completes the proof of our statement.

In order to apply and implement the inductive factorization property (2.1) one needs two steps:

(i) to renormalize all primitively divergent graphs, i.e. all divergent diagrams with no proper subdivergences, in particular, to extend all (superficially) divergent 2-point functions $G_{i j}$ to distributions on $\mathcal{S}\left(\mathbb{R}^{D}\right)$;

(ii) to extend the resulting associate homogeneous distributions defined on the complement of the full diagonal $x_{1}=x_{2}=\ldots=x_{n}$ to distributions on $\mathcal{S}\left(\mathbb{R}^{D(n-1)}\right)$.

We shall only elaborate on the first step in this exposé. Concerning step (ii), briefly reviewed in Sect. 4, we refer to our paper [NST].

A primitively divergent graph gives rise to a homogeneous distribution $G^{0}(\vec{x})$ defined on $\mathbb{R}^{N} \backslash\{0\}$ (i.e. off the small diagonal, as $\vec{x}$ is expressed in terms of the coordinate differences). The following statement concerns more generally associate homogeneous distributions and thus applies to any graph with renormalized subdivergences.

Theorem 2.2. Let $\Sigma$ be any cone section - i.e., a smooth (compact) hypersurface in $\mathbb{R}^{N} \backslash\{0\}$ that intersects transversally every ray $\{\lambda \vec{x}\}_{\lambda>0}(\vec{x} \neq 0)$ and let $\rho_{\Sigma}(\vec{x})$ be a positive smooth function such that $\vec{u}:=\rho(\vec{x})^{-1} \vec{x} \in \Sigma$. Then every associate homogeneous distribution of degree $-d$ and order $n$ has an expansion of the form ${ }^{5}$

$$
\begin{gathered}
G^{0}(r \vec{u})=\sum_{m=0}^{n} G_{m}^{\Sigma}(\vec{u}) L_{-d m}(r), \quad r=\rho_{\Sigma}(\vec{x}), \\
L_{a m}(r)=\theta(r) r^{a} \frac{(\ell n r)^{m}}{m !} \quad\left(=r^{a} \frac{(\ell n r)^{m}}{m !} \text { for } r>0\right) .
\end{gathered}
$$

\footnotetext{
${ }^{5} \mathrm{~A}$ similar decomposition in an overall scale and angle variables is derived and used very recently in momentum space in $[\mathrm{BKr}]$.
} 
The proof uses induction in $n$, based on the formula

$$
(E+d) L_{-d n}=L_{-d n-1} \text { for } E=\vec{x} \frac{\partial}{\partial \vec{x}}, n=1,2, \ldots,
$$

along with the observation that for $n=0$

$$
\frac{\partial}{\partial r}\left(r^{d} G^{0}(r \vec{u})\right)=0 .
$$

Thus the renormalization problem is reduced to the extension of 1-dimensional distributions of type (2.3). The latter is achieved by exploiting the simple pole structure of analytic regularization $[\mathrm{Sp}]$ and the resulting generating formula (see Appendix):

$$
\theta(r) r^{\epsilon-\kappa-1}-\frac{(-1)^{\kappa}}{\kappa ! \epsilon} \delta^{(\kappa)}(r)=\sum_{\kappa=0}^{\infty} L_{-\kappa-1 n}(r) \epsilon^{n} .
$$

The distributions $L_{-d n}$ can be then defined on the real line using (MC3) and (2.4); they depend on a single scale parameter hidden in the argument of the logarithm (see Appendix).

The following proposition may serve as a definition of both the notion of a residue Res and of a primary renormalization map $\mathcal{P}_{N}^{\Sigma}: \mathcal{S}^{\prime}\left(\mathbb{R}^{N} \backslash\{0\}\right) \rightarrow \mathcal{S}^{\prime}\left(\mathbb{R}^{N}\right)$.

Theorem 2.3. If $G^{0}(\vec{x})$ is a homogeneous distribution of degree $-d$ on $\mathbb{R}^{N} \backslash\{0\}$ $(d=N+\kappa \geq N)$, then

$$
\rho_{\Sigma}(\vec{x})^{\epsilon} G^{0}(\vec{x})-\frac{1}{\epsilon}(\operatorname{Res} G)(\vec{x})=G^{\Sigma}(\vec{x})+0(\epsilon) \quad\left(G^{\Sigma}=\mathcal{P}_{N}^{\Sigma} G^{0}\right) ;
$$

here $\operatorname{Res} G$ is a distribution with support at the origin whose calculation is reduced to the case $d=N$ of a logarithmically divergent graph by using the identity

$$
\operatorname{Res} G=\frac{(-1)^{\kappa}}{\kappa !} \partial_{i_{1}} \ldots \partial_{i_{\kappa}}\left(\operatorname{Res}\left(x^{i_{1}} \ldots x^{i_{\kappa}} G\right)\right)(\vec{x})
$$

where summation is understood over all repeated indices $i_{1}, \ldots, i_{\kappa}$ from 1 to $N$. If $G^{0}(\vec{x})$ is homogeneous of degree $-N$ then

$$
\operatorname{Res} G(\vec{x})=\left(\operatorname{res} G^{0}\right) \delta(\vec{x}) \quad\left(\text { for }(E+N) G^{0}(\vec{x})=0\right)
$$

where

$$
\operatorname{res} G^{0}=\int_{\Sigma} G^{0}(\vec{x}) \sum_{j=1}^{N}(-1)^{j-1} x^{j} d x^{1} \wedge \ldots d \widehat{x}^{j} \ldots \wedge d x^{N}
$$

is independent of $\Sigma$ since the form under the integral sign is closed. (A hat, $\widehat{ }$, over an argument means, as usual, that this argument is omitted.)

Proof. The fact that the distribution valued function of $\epsilon \rho_{\Sigma}^{\epsilon} G^{0}$ is meromorphic and only has a simple pole at $\epsilon=0$ follows from Theorem 2.2 and Eq. (2.5). 
Eq. (2.7) follows from the assumed homogeneity property $\partial_{i} x^{i} G^{0}=-\kappa G^{0}$ of $G^{0}$. The integrand in (2.9) is a contraction of $G^{0} \mathrm{Vol}$ with the Euler vector field:

$$
i_{E} G^{0} \mathrm{Vol}=\sum_{j=1}^{N} G^{0}(-1)^{j} x^{j} d x^{1} \wedge \ldots d \widehat{x}^{j} \ldots \wedge d x^{N}
$$

and it is a (homogeneous) form of maximal degree in the $(N-1)$-dimensional projective space for $\lambda^{N} G^{0}(\lambda \vec{x})=G^{0}(\vec{x})$.

The residue (2.9) is a special case of the so called Wodzicki residue (see [G-B] $[\mathrm{G}-\mathrm{BVF}]$ and references therein). 


\section{Residues and renormalization of primitively divergent graphs}

For the (euclidean covariant) 2-point function in a $D$-dimensional space-time $N=D\left(\vec{x}=x=x_{1}-x_{2}\right)$ it is natural to choose for $\Sigma$ the unit hypersphere $\mathbb{S}^{D-1}$, so that $\rho_{\Sigma}(x)=\sqrt{x^{2}}=: r$. For a scalar 2-point function of a composite field of dimension $\frac{D}{2}$ ( $D$-even), we would have

$$
G^{0}(x)=\frac{C}{\left(x^{2}\right)^{D / 2}}, \quad \operatorname{Res} G=C\left|\mathbb{S}^{D-1}\right| \delta(x)
$$

where $\left|\mathbb{S}^{2 m-1}\right|=\frac{2 \pi^{m}}{(m-1) !}$.

The renormalization map $\mathcal{R}_{D}^{\Sigma}: G^{0} \rightarrow G^{\Sigma}$ (2.6) can be computed explicitly in terms of the radial coordinate $r$ of Eq. (2.5) (see Appendix).

Here we shall compute it instead in Cartesian coordinates in two examples of 4-dimensional $(4 D)$ scalar field theory.

Example 3.1. The logarithmically divergent 2-point graph shown on Fig. 1a

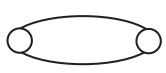

$1 \mathrm{a}$

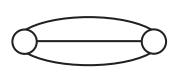

$1 \mathrm{~b}$

Figure 1.

Logarithmically and quadratically divergent 2-point graphs.

is ubiquitous as a (sub)divergence in any scalar field theory in $4 D$ : it appears as a self-energy graph in a $\varphi^{3}$ model and as a contribution to the 4-particle scattering amplitude in the $\varphi^{4}$ theory. The limit $\epsilon \rightarrow 0$ in (2.6) for this 1-loop graph reads

$$
\begin{aligned}
G_{1}(x, \ell)= & \lim _{\varepsilon \rightarrow 0}\left[\frac{1}{\left(x^{2}\right)^{2}}\left(\frac{x^{2}}{\ell^{2}}\right)^{\varepsilon}-\frac{2 \pi^{2}}{2 \varepsilon} \delta(x)\right] \\
= & \frac{1}{2} \frac{\partial}{\partial x^{\alpha}}\left[\frac{x^{\alpha}}{\left(x^{2}\right)^{2}} \ln \left(\frac{x^{2}}{\ell^{2}}\right)\right]\left(=\frac{1}{r^{2}} \frac{\partial}{\partial r^{2}}\left(\ln \frac{r^{2}}{\ell^{2}}\right)_{+},\right. \\
& (\ln \rho)_{+}=\left\{\begin{array}{ccc}
\ln \rho & \text { for } & \rho>0 \\
0 & \text { for } & \rho<0
\end{array}\right) .
\end{aligned}
$$

This is another instance of differential renormalization (cf. Eq. (A.4) and see [FJL], [HL], [Pr]). Renormalized expressions of the type $\frac{\partial}{\partial x^{\alpha}}\left[\frac{x^{\alpha}}{\left(x^{2}\right)^{2}} \ell n \frac{x^{2}}{\ell^{2}}\right]$ (sum over $\alpha$ ) are used systematically in [G-B].

Remark 3.1. Note that the double and the triple lines in Fig. 1 should both be viewed as a single line with a different decoration (corresponding to different 
powers, $\mu=2$ and $\mu=3$, in (1.1)). Thus, the self-energy graph on Fig. 1b, which displays overlapping divergences in momentum space, is primitively divergent in $x$-space according to our definition. Its renormalized expression is additionally restricted by the requirement of full euclidean invariance. (In general, we require the presence of as much of the symmetry of the rational function in the renormalized expression as allowed by the existing anomalies.) Applying further requirement (MC3) which yields the identity $G_{1}(x, \ell)=x^{2} G_{2}(x, \ell)$, valid for the original rational functions away from the origin, we find

$$
\begin{aligned}
G_{2}(x, \ell) & =\lim _{\varepsilon \rightarrow 0}\left\{\frac{1}{\left(x^{2}\right)^{3}}\left(\frac{x^{2}}{\ell^{2}}\right)^{\varepsilon}-\frac{\pi^{2}}{8 \varepsilon} \Delta \delta(x)\right\} \\
& =\frac{3 \pi^{2}}{16} \Delta \delta(x)+\frac{\Delta}{8} G_{1}(x, \ell) .
\end{aligned}
$$

In deriving (3.3) we have used the identities

$$
\begin{gathered}
\Delta f=4 \frac{\partial^{2}}{\partial \rho^{2}}(\rho f)+\frac{1}{\rho} \Delta_{\omega} f \quad \text { for } \rho=x^{2}\left(=r^{2}\right), x=r \omega \\
\frac{1}{\rho^{n+1}}\left(\frac{\rho}{\ell^{2}}\right)^{\varepsilon}=\frac{1}{(n-\varepsilon)(n-1-\varepsilon)^{2} \ldots(1-\varepsilon)^{2}(-\varepsilon)}\left(\frac{\partial^{2}}{\partial \rho^{2}} \rho\right)^{n} \frac{1}{\rho}\left(\frac{\rho}{\ell^{2}}\right)^{\varepsilon} \\
=\frac{1}{n !(n-1) !}\left(\frac{\Delta}{4}\right)^{n-1}\left(\frac{\pi^{2}}{\varepsilon} \delta(x)+\pi^{2} s_{n} \delta(x)+G_{1}(x, \ell)\right)+O(\varepsilon),
\end{gathered}
$$

where $s_{n}$ is a sum of partial harmonic series (cf. (A.5)):

$$
s_{n}=\sum_{j=1}^{n-1} \frac{1}{j}+\sum_{j=2}^{n} \frac{1}{j} \quad\left(s_{1}=0, s_{2}=\frac{3}{2}, s_{3}=\frac{7}{3}, \ldots\right) .
$$

One can use a more general (homogeneous, $O(D)$-invariant) norm on the distances $x_{i j}^{2}$ instead of the $(O(N)$-invariant) radial coordinate for $N=D(n-1)$ in order to compute both the residue and the renormalized expression of a primitively divergent graph as illustrated on the following n-loop example.

Example 3.2. We consider the $4 D n$-loop $(n+1$-point $)$ primitively divergent Feynman amplitude

$$
G_{n}=\left(\prod_{i=1}^{n} x_{0 i}^{2} x_{i i+1}^{2}\right)^{-1}, x_{n+1} \equiv x_{1},
$$

which we shall parametrize by the spherical coordinates of the $n$ independent 4 -vectors $x_{0 i}$ :

$$
x_{0 i}=r_{i} \omega_{i}, \quad r_{i} \geq 0, \quad \omega_{i}^{2}=1, \quad i=1,2, \ldots, n .
$$

An important special case is given by the complete 4-point graph on Fig. 2 


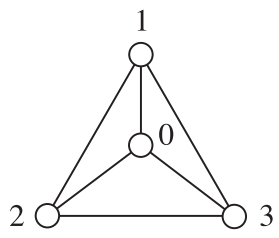

$$
G_{3}=\frac{1}{x_{01}^{2} x_{02}^{2} x_{03}^{2} x_{12}^{2} x_{23}^{2} x_{13}^{2}}
$$

Figure 2.

The tetrahedron graph in the $\left(\varphi^{4}\right)_{4}$-theory.

Setting 6

$$
G_{n}^{\varepsilon}=\left(\frac{R^{2}}{\ell^{2}}\right)^{\varepsilon} G_{n}, R=\max \left(r_{1}, \ldots, r_{n}\right),
$$

we shall compute its residue by first integrating the corresponding analytically regularized density $G_{n}^{\varepsilon}$ Vol over the angles $\omega_{i}$ using the identification of the propagators $\frac{1}{x_{i j}^{2}}$ with the generating functions for the Gegenbauer polynomials. Having in mind applications to a scalar field theory in $D$ dimensions (see Example 4.2 below) we shall write down the corresponding more general formulas. The propagator $\left(x_{12}^{2}\right)^{-\lambda}$ of a free massless scalar field in $D=2 \lambda+2$ dimensional space-time is expanded as follows in (hyperspherical) Gegenbauer polynomials:

$$
\begin{gathered}
\left(x_{i j}^{2}\right)^{-\lambda}=\left(r_{i}^{2}+r_{j}^{2}-2 r_{i} r_{j} \omega_{i} \omega_{j}\right)^{-\lambda}=\frac{1}{R_{i j}^{2 \lambda}} \sum_{n=0}^{\infty}\left(\frac{r_{i j}}{R_{i j}}\right)^{n} C_{n}^{\lambda}\left(\omega_{i} \omega_{j}\right), \\
R_{i j}=\max \left(r_{i}, r_{j}\right), \quad r_{i j}=\min \left(r_{i}, r_{j}\right), \quad i \neq j, i, j=1,2,3 .
\end{gathered}
$$

We shall also use the integral formula

$$
\int_{\mathbb{S}^{2 \lambda+1}} d \omega C_{m}^{\lambda}\left(\omega_{1} \omega\right) C_{n}^{\lambda}\left(\omega_{2} \omega\right)=\frac{\lambda\left|\mathbb{S}^{2 \lambda+1}\right|}{n+\lambda} \delta_{m n} C_{n}^{\lambda}\left(\omega_{1} \omega_{2}\right),
$$

where $\left|\mathbb{S}^{2 \lambda+1}\right|=\frac{2 \pi^{\lambda+1}}{\Gamma(\lambda+1)}$ is the volume of the unit hypersphere in $D=2 \lambda+2$ dimensions.

Clearly, the expansion (3.7) requires an ordering of the lengths $r_{i}$. In general, one should consider separately $n$ ! sectors, obtained from one of them, say

$$
r_{1} \geq r_{2} \geq \ldots \geq r_{n}(\geq 0)
$$

by permutations of the indices. It is, in fact, sufficient to consider just the sector (3.9) (and multiply the result for the residue by $n$ !). (Because of the symmetry

\footnotetext{
${ }^{6}$ The fact that the maximum function $R$, which replaces $\rho_{\Sigma}(\vec{x})$ of Theorem 2.2 , does not depend smoothly on the coordinates, requires, in general, a special treatment of the lower dimensional manifolds of discontinuities of its derivatives. (See Example 4.1 below.)
} 
of the tetrahedron graph (Fig. 2) this is obvious for $n=3$ but it is actually true for any $n(\geq 3)$.) The result involves a polylogarithmic function:

$$
\begin{aligned}
\widetilde{G}_{n}^{\varepsilon}:= & \int_{\mathbb{S}^{3}} \ldots \int_{\mathbb{S}^{3}} G_{n}^{\varepsilon}\left(r_{1} \omega_{1}, \ldots, r_{n} \omega_{n}\right) \mathrm{Vol} \\
= & \left(2 \pi^{2}\right)^{n}\left(\frac{r_{1}}{\ell}\right)^{2 \varepsilon} \frac{d r_{1} \wedge \ldots \wedge d r_{n}}{r_{1} \ldots r_{n}} L i_{n-2}\left(\frac{r_{n}^{2}}{r_{1}^{2}}\right), \\
& L i_{n-2}(\xi)=\sum_{m=1}^{\infty} \frac{1}{m^{n-2}} \xi^{m}\left(\xi=\frac{r_{n}^{2}}{r_{1}^{2}}\right)
\end{aligned}
$$

$\left(r_{n}=\min \left(r_{1}, \ldots, r_{n}\right), r_{1}=\max \left(r_{1}, \ldots, r_{n}\right)(=R)\right)$. To derive the last equation we have applied once more (3.8) and used

$$
\left(C_{m}^{1}\left(\omega_{1}^{2}\right)=\right) C_{m}^{1}(1)=m+1 .
$$

The residue distribution corresponding to the (integrated over the angles) density (3.10) is given by

$$
\operatorname{Res} \widetilde{G}_{n}^{\varepsilon}=\operatorname{res} G_{n}^{0} \delta\left(r_{1}\right) \ldots \delta\left(r_{n}\right) d r_{1} \wedge \ldots \wedge d r_{n}
$$

where

$$
\begin{gathered}
\operatorname{res} G_{n}^{0}=n ! \lim _{\varepsilon \rightarrow 0} 2 \varepsilon \int_{r_{1}=0}^{\infty} \int_{r_{2}=0}^{r_{1}} \ldots \int_{r_{n}=0}^{r_{n-1}} \widetilde{G}_{n}^{\varepsilon}=n !\left(2 \pi^{2}\right)^{n} \int_{K_{n-1}} \ldots \int \omega, \\
\omega:=L i_{n-2}(\xi) \frac{r_{1} d r_{2} \wedge \ldots \wedge d r_{n}-r_{2} d r_{1} \wedge d r_{3} \ldots \wedge d r_{n}+\ldots(-1)^{n-1} r_{n} d r_{1} \wedge \ldots \wedge d r_{n-1}}{r_{1} \ldots r_{n}} \\
(d \omega=0) .
\end{gathered}
$$

Here $\omega$ is a closed homogeneous form on the compact projective cone

$$
K_{n-1}=\left\{\left(r_{1}, \ldots, r_{n}\right) \in \mathbb{P}_{n-1} ; r_{i} \geq 0\left(\sum_{i=1}^{n} r_{i}>0\right)\right\} .
$$

The integration in (3.12) may be performed over any transverse surface. Choosing $R\left(=r_{1}\right)=1$ we find

$$
\begin{aligned}
\operatorname{res} G_{n}^{0} & =n !\left(2 \pi^{2}\right)^{n} \int_{0}^{1} \frac{d r_{2}}{r_{2}} \ldots \int_{0}^{r_{n-1}} \frac{d r_{n}}{r_{n}} L i_{n-2}\left(r_{n}^{2}\right) \\
& =n ! 2 \pi^{2 n} \zeta(2 n-3) .
\end{aligned}
$$

In particular, for the tetrahedron graph, $n=3$, we reproduce the known result, $\operatorname{res} G_{3}^{0}=12 \pi^{6} \zeta(3)$ - see, for instance, [G-B].

The integration technique based on the properties of Gegenbauer polynomials has been introduced in the study of $x$-space Feynman integrals in [CKT]. The appearance of $\zeta$-values in similar computations has been detected in early work of Rosner $[\mathrm{R}]$ and Usyukina $[\mathrm{U}]$. It was related to the non-trivial topology of graphs by Broadhurst and Kreimer [BrK], [Kr]. 


\section{Dilation anomaly. Examples of graphs with subdivergences}

We now turn to the behaviour under dilations of a renormalized primitively divergent density $G(\vec{x}) \mathrm{Vol}$ of index $\kappa(\geq 0)$. By the definition of $G$ Vol the dilation anomaly

$$
A(\vec{x}, \lambda):=\lambda^{\kappa} G(\lambda \vec{x}) \mathrm{Vol}-G(\vec{x}) \mathrm{Vol}
$$

is a distribution valued density with support at the small diagonal, $x_{1}=x_{2}=$ $\ldots=x_{n}$. Invoking the requirement (MC2), we can restrict it, following $[\mathrm{H}]$, by demanding that it is again homogeneous in $\vec{x}$ of degree $-\kappa$ :

$$
A(\vec{x}, \lambda)=\sum_{\boldsymbol{\alpha},|\boldsymbol{\alpha}|=\kappa} a_{\boldsymbol{\alpha}}(\lambda) D_{\boldsymbol{\alpha}} \delta(\vec{x}) \prod_{i=1}^{n-1} d^{D} x_{i n}
$$

where $\delta(\vec{x})$ is the $D(n-1)$-dimensional $\delta$-function,

$$
D_{\boldsymbol{\alpha}}=\prod_{i=1}^{n-1} \prod_{\nu=1}^{D}\left(\partial_{i}^{\nu}\right)^{\alpha_{i \nu}}, \quad|\boldsymbol{\alpha}|=\sum_{i, \nu} \alpha_{i \nu} .
$$

Repeated application of the dilation law (4.1) yields the cocycle condition ${ }^{7}$

$$
a_{\boldsymbol{\alpha}}(\lambda \mu)=a_{\boldsymbol{\alpha}}(\lambda)+a_{\boldsymbol{\alpha}}(\mu) .
$$

The general form of $a_{\boldsymbol{\alpha}}$ satisfying (4.3) is

$$
a_{\boldsymbol{\alpha}}(\lambda)=a_{\boldsymbol{\alpha}}(G) \ln \lambda
$$

where $a_{\boldsymbol{\alpha}}(G)$ is a linear functional of the Green function $G$ (or the corresponding density $G \mathrm{Vol})$. It is important to note that the coefficient $a_{\boldsymbol{\alpha}}(G)$ in (4.4) is independent of the ambiguity in the definition of the renormalized Green function. Once the problem of renormalizing a primitively divergent graph is reduced to a 1-dimensional one (as in Sect. 2) this follows from the simple observation that the coefficient of $\ell n r$ in (A.5) is independent of the ambiguity reflected in the scale parameter $\ell$ (and of the transverse hypersurface $\Sigma$ that enters (2.9)).

In fact, each renormalization of a subdivergence in a given graph increases by one the order - i.e. the maximal power of $\ell n \lambda$ in the associate homogeneity law. Since $r \frac{\partial}{\partial r}(\ell n r)^{j}=j(\ell n r)^{j-1}$, a general associate homogeneous renormalized Feynman amplitude $G$ will satisfy Eq. $(1.7),(E+d)^{k+1} G(\vec{x})=0$. We can then characterize $G$ by a (column) vector $\boldsymbol{G}=\left(G_{0}=G, G_{1}=(E+d) G_{0}, \ldots, G_{k}=\right.$ $\left.(E+d) G_{k-1}\right)$ of distributions. It carries an indecomposable representation of the dilation group ${ }^{8}$ of degree $-d$ and order $k$ such that

$$
\boldsymbol{G}(\vec{x}) \rightarrow \lambda^{d} \boldsymbol{G}(\lambda \vec{x})=e^{\Delta \ell n \lambda} \boldsymbol{G}(\vec{x})=\sum_{j=0}^{k} \frac{(\ell n \lambda)^{j}}{j !} G_{j}(\vec{x})
$$

\footnotetext{
${ }^{7}$ Usually, in perturbation theory one is dealing with Lie algebra cohomology. Group cohomology has occurred in various contexts in the early 1980's [S82/93] [F].

${ }^{8}$ Representations of this type have been considered back in the 1970's [FGG] within a study of a spontaneous breaking of dilation symmetry.
} 
where $\Delta$ is a nilpotent Jordan cell with $k$ units above the diagonal. The nilpotency condition $\Delta^{k+1}=0$ remains invariant under an arbitrary non-singular transformation $\boldsymbol{G} \rightarrow S \boldsymbol{G}, \Delta \rightarrow S^{-1} \Delta S$. One usually only uses this freedom to change the relative normalization of $G_{j}$.

It follows from the factorization property (2.1) that the dimension of the support of $G_{j}$ is decreasing with $j$ and

$$
G_{k}(\vec{x})=(\vec{x} \vec{\partial}+d) G_{0}(\vec{x})=\sum_{\alpha} a_{\alpha}(G) D_{\alpha} \delta(x) .
$$

Following the terminology of Gelfand-Shilov [GS] we call both $\boldsymbol{G}$ and its components associate homogeneous distributions (cf. (1.7)).

The following simple example of a graph with a subdivergence illustrate the complication (mentioned in connection with Eq. (3.6)) coming from the use of a non-smooth radial coordinate.

Example 4.1. Renormalization the 3-point two loop diagram displayed on Fig. 3.

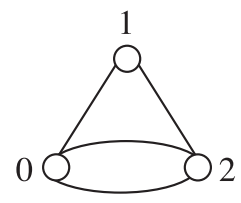

$$
G_{\Delta}=\frac{1}{x_{01}^{2} x_{12}^{2}\left[x_{02}^{2}\right]^{2}}
$$

Figure 3.

Logarithmically divergent 3-point graph with a 2-point subdivergence.

We introduce as independent variables the spherical coordinates of the vectors $x_{0 i}, i=1,2$

$$
x_{01}=r \omega_{1}, \quad x_{02}=\rho \omega_{2}, \quad r, \rho \geq 0, \quad \omega_{i}^{2}=1\left(\text { i.e. } \omega_{i} \in \mathbb{S}^{3}\right) i=1,2
$$

and set

$$
\omega_{1} \cdot \omega_{2}=\cos \vartheta, \quad x_{12}^{2}=r^{2}+\rho^{2}-2 r \rho \cos \vartheta .
$$

The renormalized 2-point Green function (3.2), corresponding to the subgraph of vertices $(0,2)$ is

$$
G_{1}\left(x_{02}, \ell\right)=\frac{1}{2} \frac{\partial}{\partial x_{02}^{\alpha}}\left[\frac{x_{02}^{\alpha}}{\left(x_{02}^{2}\right)^{2}} \ln \frac{x_{02}^{2}}{\ell^{2}}\right]_{+}=\frac{1}{\rho^{3}} \frac{\partial}{\partial \rho}\left(\ln \frac{\rho}{\ell}\right)_{+} .
$$

(The last expression only makes sense as a density after multiplying with the volume element $d^{4} x=\rho^{3} d \rho d^{3} \omega$ that cancels the $\frac{1}{\rho^{3}}$ factor and permits to transfer the derivative to the test function.) 
Next we shall write down the density $G_{\Delta}$ Vol with renormalized subdivergence integrated over the six angular variables $\omega_{1}$ and $\omega_{2}$

$$
\begin{aligned}
G_{\Delta} \mathrm{Vol} & :=\left[\int d^{3} \omega_{1} \int d^{3} \omega_{2} G_{\Delta}\left(r \omega_{1}, \rho \omega_{2} ; \ell\right)\right] r^{3} d r \rho^{3} d \rho \\
& =8 \pi^{3} \int_{0}^{\pi} \frac{\sin ^{2} \vartheta d \vartheta}{r^{2}+\rho^{2}-2 r \rho \cos \vartheta} \frac{\partial}{\partial \rho}\left(\ell n \frac{\rho}{\ell}\right)_{+} r d r d \rho \\
& =4 \pi^{4} \frac{r d r d \rho}{r_{\vee}^{2}} \frac{\partial}{\partial \rho}\left(\ell n \frac{\rho}{\ell}\right)_{+}, r_{\vee}=\max (r, \rho)=\frac{r+\rho+|r-\rho|}{2} .
\end{aligned}
$$

Smearing $G_{\Delta}$ Vol with a test function $f(r, \rho)$ we find that the leading term, $L T G_{\Delta} \mathrm{Vol}$, for $r_{\vee} \rightarrow 0$ (the only one that requires overall renormalization) corresponds to $r=\rho$

$$
\left(L T G_{\Delta}^{R} \operatorname{Vol}, f\right)=-4 \pi^{4} \int_{0}^{\infty} d r \frac{\ell n^{2}\left(\frac{r}{\ell}\right)}{2} \frac{d}{d r} f(r, r) .
$$

Here we have made use of the renormalized associate homogeneous distribution $L_{-11}(r)$ thus illustrating Theorem 2.2.

Somewhat symbolically we can write

$$
G_{\Delta}^{R}(r, \rho ; \ell) \operatorname{Vol}=4 \pi^{4} L_{-11}\left(\frac{r}{\ell}\right) \delta(\rho-r) \frac{d r}{\ell} d \rho+G_{0}(r, \rho) \operatorname{Vol} L_{01}\left(\frac{\rho}{\ell}\right) d \rho
$$

where $G_{0} \mathrm{Vol}$ is the regular part of the homogeneous 1-form $4 \pi^{4} \frac{r d r}{r_{v}^{2}}($ for $\rho \neq r)$.

Displaying the associate homogeneity law for the renormalized density (4.12) we observe a manifestation of the general rule: only the coefficient of the highest $\log$ term ( $\ell n \lambda$ for $L_{01} d \rho$ and $(\ell n \lambda)^{2}$ for $\left.L_{-11} d r\right)$ is independent of the ambiguity parametrized here by the scale $\ell$ in the renormalized subdivergence.

Remark 4.1. One could be tempted to replace the renormalization parameter $\ell$ in the expression (4.10) by the (external to the divergent 2-point subgraph) variable $r$ for $r>\rho$. This would amount to subtracting a local in $\rho$ term, $4 \pi^{4} \frac{d r}{r} \ln \frac{r}{\ell} \delta(\rho) d \rho$. It is straightforward to observe, however, that neglecting such a term in (4.10) would violate the causal factorization requirement (2.1).

The techniques developed in Example 4.1 also apply to more complicated graphs ( $c f$. Example 3.2 in [NST]).

Example 4.2. As a last example we consider the graph displayed on Fig. 4 


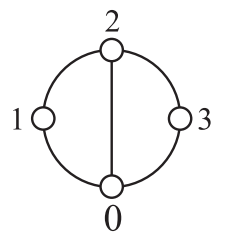

$$
\begin{aligned}
x_{0 j} & =r_{j} \omega_{j}, r_{j} \geq 0, \omega_{j} \in \mathbb{S}^{5} \\
G_{\mathbb{O}} & =\left(r_{1}^{2} r_{2}^{2} r_{3}^{2} x_{12}^{2} x_{23}^{2}\right)^{-2}
\end{aligned}
$$

Figure 4.

Quadratically divergent diagram in 6-dimensions.

which exhibits overlapping divergences in 6-dimensional space-time.

Applying the relations (3.7) (3.8) for $\lambda=2$, we find the following expression for the analytically regularized integrated with respect to the angles Green function density

$$
\widetilde{G}_{\mathbb{O}}^{\varepsilon_{1} \varepsilon_{2}}=\pi^{9} \frac{r_{1} r_{2} r_{3}}{\left(R_{12} R_{23}\right)^{4}}\left(\frac{R_{12}^{2}}{\ell_{1}^{2}}\right)^{\varepsilon_{1}}\left(\frac{R_{23}^{2}}{\ell_{2}^{2}}\right)^{\varepsilon_{2}} d r_{1} d r_{2} d r_{3},
$$

where $R_{i j}=\max \left(r_{i}, r_{j}\right)\left(c f\right.$. (3.7)). The renormalized expression for $G_{\mathbb{O}}$ again depends, as in the preceding examples (see, in particular, Example 3.2) on the inequalities satisfied by the radial variables. For

$$
r_{1}<r_{2}<r_{3}
$$

(and, similarly, for $r_{3}<r_{2}<r_{1}$ ) we have a case of nested singularities. One first renormalizes the logarithmicly divergent triangular subgraph with vertices $(0,1,2)$. Integrating first with respect to $r_{1}$ in the domain (4.14) we find

$$
\begin{aligned}
& \lim _{\varepsilon_{1} \rightarrow 0}\left(\int_{0}^{r_{2}} \widetilde{G}_{\mathbb{O}}^{\varepsilon_{1} \varepsilon_{2}}-\frac{\pi^{9}}{4 \varepsilon_{1}} \delta\left(r_{2}\right)\left(\frac{r_{3}}{\ell_{2}}\right)^{2 \varepsilon_{2}} \frac{d r_{2} d r_{3}}{r_{3}^{3}}\right) \\
= & \frac{\pi^{9}}{2} d\left(\ln \frac{r_{2}}{\ell_{1}}\right)\left(\frac{r_{3}}{\ell_{2}}\right)^{2 \varepsilon_{2}} \frac{d r_{3}}{r_{3}^{3}} .
\end{aligned}
$$

The renormalization of the resulting quadratically divergent in $r_{3}$ associate homogeneous distribution follows the lines of Example 4.1. The case $r_{1}<r_{2}>r_{3}$, in which $R_{12}=R_{23}=r_{2}$ and "the divergences overlap", is actually simpler; it is reduced to a single radial renormalization. Setting $\varepsilon_{1}+\varepsilon_{2}=\frac{\varepsilon}{2}$ and $\ell_{1} \ell_{2}=\ell^{2}$ and integrating in $r_{1}$ and $r_{3}$, we find

$$
\lim _{\varepsilon \rightarrow 0}\left(\int_{r_{1}=0}^{r_{2}} \int_{r_{3}=0}^{r_{2}} G_{\mathbb{O}}^{\varepsilon}-\frac{\pi^{9}}{8} \frac{\delta^{\prime \prime}\left(r_{2}\right)}{2 \varepsilon} d r_{2}\right)=\frac{\pi^{9}}{8}\left(\frac{d^{3}}{d r^{3}} \ln \frac{r}{\ell}+\frac{3}{2} \delta^{\prime \prime}(r)\right) .
$$

\section{Concluding remarks}

The work [NST], surveyed here, is concerned with a mathematical reformulation of the poblem of ultraviolet renormalization of massless QFT. The extension of rational homogeneous functions to associate homogeneous distributions of 
the same degree obeying (euclidean) factorization, considered here, only partly resolves the physical problem (see $[\mathrm{N}]$ ). It does not consider integration over internal vertices in concrete Lagrangian theories (like $\varphi^{4}$ ) and so does not control the corresponding adiabatic limit (which is separated in standard approaches from the study of on shell infrared singularities ${ }^{9}$ ).

The present survey is only confined to the part of [NST] dealing with the euclidean picture. The reader willing to understand the physical origin of the causal factorization and the way one goes around the light cone singularities should consult the original paper.

Acknowledgments. The authors thank Detlev Buchholz, Maxim Kontsevich and Dirk Kreimer for discussions. N.M.N. and I.T. acknowledge partial support by grant DO 02-257 of the Bulgarian National Science Foundation. I.T. thanks the organizers of the International Workshops LT-9 and SQS'2011 for the invitation and the IHÉS and the Theory Division at CERN for hospitality during the course of this work.

\section{Appendix A. Radial associate homogeneous dis- tributions}

The study of radial homogeneous distributions is based on the observation $([\mathrm{H}]$ Sect. 3.2) that the family of distributions ("devided powers")

$$
\chi^{a}(r):=\frac{\left(r^{a}\right)_{+}}{\Gamma(a+1)}, \quad a \neq-1,-2, \ldots \quad\left(\left(r^{a}\right)_{+} \equiv \theta(r) r^{a}\right)
$$

is uniquely extendable to a distribution valued entire analytic function in $a$. The property $\Gamma(a+1)=a \Gamma(a)$ gives

$$
\frac{d}{d r} \chi^{a}(r)=\chi^{a-1}(r) \quad\left(r \chi^{a}(r)=(a+1) \chi^{a+1}(r)\right) .
$$

Combined with $\chi^{0}(r)=\theta(r)$ the (Heaviside) characteristic function of the positive semiaxis - we find

$$
\chi^{-\kappa-1}(r)=\delta^{(\kappa)}(r), \quad \kappa=0,1, \ldots\left(\int \delta^{(\kappa)}(r) f(r) d r=(-1)^{\kappa} f^{(\kappa)}(0)\right) .
$$

From the known pole structure of $\Gamma(a)$ we deduce the formula (2.5) for the generating function of $L_{-\kappa-1 n}$. The distributions $L_{-\kappa-1 n}$ can be defined in terms of differential renormalization [FJL]:

$$
\begin{aligned}
L_{-\kappa-1 n}(r) & =\lim _{\epsilon \rightarrow 0} \frac{1}{n !} \frac{\partial^{n}}{\partial \epsilon^{n}}\left(\theta(r) r^{\epsilon-\kappa-1}-\frac{\delta^{(\kappa)}(-r)}{\epsilon \kappa !}\right) \\
& =\frac{(-1)^{\kappa}}{\kappa !}\left(\frac{d}{d r}\right)^{\kappa+1} \sum_{m=0}^{n+1} \sigma_{\kappa m} L_{0 n+1-m},
\end{aligned}
$$

\footnotetext{
${ }^{9}$ We thank Detlev Buchholz for stressing this point to us.
} 
where $L_{0 \nu}(r)=\theta(r) \frac{(\ell n r)^{\nu}}{\nu !}$ are (integrable) powers of logarithms and the constants $\sigma_{\kappa m}$ are given by

$$
\begin{gathered}
\sigma_{\kappa 0}=1, \quad \sigma_{0 m}=0 \quad \text { for } \quad m=1, \ldots, n+1, \\
\sigma_{\kappa m}=\sigma_{\kappa-1 m}+\frac{\sigma_{\kappa m-1}}{\kappa}=\sum_{1 \leq j_{1} \leq \ldots \leq j_{m} \leq \kappa} \frac{1}{j_{1} \ldots j_{m}} .
\end{gathered}
$$

The freedom in the extension of the rational homogeneous function $r^{-k}$ from the positive semiaxis to an associate homogeneous distribution on $\mathbb{R}$ is hidden in the scale of $\log r$. In fact, the general associate homogeneous distribution that coincides with $r^{-\kappa-1}$ for $r>0$ involves a single scale parameter $\ell$ :

$$
\begin{aligned}
\ell^{-\kappa-1} L_{-\kappa-1,0}\left(\frac{r}{\ell}\right) & =\frac{(-1)^{\kappa}}{\kappa !}\left\{\frac{d^{\kappa+1}}{d r^{\kappa+1}}\left(\theta(r) \ell n \frac{r}{\ell}\right)+\sum_{j=1}^{\kappa} \frac{1}{j} \delta^{(\kappa)}(r)\right\} \\
& =L_{-\kappa-1,0}(r)-\ln \ell \frac{\delta^{(\kappa)}(-r)}{\kappa !} .
\end{aligned}
$$

Once $\ell$ is fixed, say $\ell=1$, all distributions $L_{k n}(r)(k \in \mathbb{Z}, n=0,1, \ldots)$ are uniquely determined.

Proposition A.1. The distributions $L_{k n}(r)$, given for negative integer $k$ by (A.4), satisfy

(i) $L_{k n}(r)=\theta(r) r^{k} \frac{(\ell n r)^{n}}{n !}$ for $r \neq 0$;

(ii) $(E-k) L_{k n}(r)=L_{k n-1}(r)$ for $n=1,2, \ldots,(E-k) L_{k 0}(r)=0$;

(iii) $r L_{k n}(r)=L_{k+1 n}(r)$.

Conversely, the properties (i) and (ii) determine uniquely the system of distributions $L_{k n}$.

Proof. Properties (i)-(iii) follow from the corresponding properties of $\theta(r) r^{\epsilon+k}$ (and from Eq. (2.5)). To prove the uniqueness, assume that there are two sets of associate homogeneous distributions $L_{k n}$ and $L_{k n}^{\prime}$ satisfying (i) and (ii). Then their differences $D_{k n}:=L_{k n}-L_{k n}^{\prime}$ would satisfy $D_{k n}=0$ for $k \geq 0$ and $D_{-\kappa-1 n}(r)=C_{\kappa n} \delta^{(\kappa)}(r)$ for $\kappa, n=0,1, \ldots$. It then follows from (ii) that

$$
0=(E+\kappa+1) C_{\kappa n+1} \delta^{(\kappa)}(r)=C_{\kappa n} \delta^{(\kappa)}(r),
$$

hence $C_{\kappa n}=0$ for all $n \geq 0$. 


\section{References}

[B] C. Bergbauer, Notes on Feynman integrals and renormalization, arXiv: 1005.3960 [hep-th].

[BBK] C. Bergbauer, R. Brunetti, D. Kreimer, Renormalization and resolution of singularities, arXiv:0908.0633v2 [hep-th].

[BEK] S. Bloch, H. Esnault, D. Kreimer, On motives and graph polynomials, Commun. Math. Phys. 267 (2006) 181-225; math/0510011.

[BK] S. Bloch, D. Kreimer, Mixed Hodge structures and renormalization in physics, Commun. Number Theory Phys. 2 (2008) 637-718, arXiv:0804.4399 [hep-th]; Feynman amplitudes and Landau singularities for 1-loop graphs, arXiv:1007.0338 [hep-th].

[BP] N.N. Bogoliubov, O. S. Parasiuk, Über die Multiplikation der Kausalfunktionen in der Quantentheorie der Felder, Acta Mathematica 97 (1957) 227266; O.S. Parasiuk, On the theory of Bogoliubov's R-operation (Russian), Ukr. Math. Zh. 12 (1960) 287-307.

[BS] N.N. Bogoliubov, D.V. Shirkov, Introduction to the Theory of Quantized Fields, 3d edition, Wiley, 1980.

[BrK] D.J. Broadhurst, D. Kreimer, Association of multiple zeta values with positive knots via Feynman diagrams up to 9 loops, Phys. Lett. B393 (1997) 403-412; hep-th/9609128.

[BKr] F. Brown, D. Kreimer, Angles, scales and parametric renormalization, arXiv:1112.1180 [hep-th].

[BF] R. Brunetti, K. Fredenhagen, Microlocal analysis and interacting quantum field theories: Renormalization on physical backgrounds, Commun. Math. Phys. 208 (2000) 623-661; math-ph/990328.

[BFV] R. Brunetti, K. Fredenhagen, R. Verch, The generally covariant locality principle: a new paradigm for local quantum physics, Commun. Math. Phys. 237 (2003) 31-68; math-ph/0112041.

[CM] O. Ceyhan, M. Marcolli, Feynman integrals and motives of configuration space, arXiv:1012.5485 [math-ph].

[CKT] K.G. Chetyrkin, A.L. Kataev, F.V. Tkachov, New approach to evaluation of multiloop Feynman integarls: the Gegenbauer polynomial x-space technique, Nucl. Phys. B174 (1980) 345-377.

[CK] A. Connes, D. Kreimer, Renormalization in quantum field theory and the Riemann-Hilbert problem I: the Hopf algebra structure of graphs and the main theorem, II: the $\beta$-function diffeomorphism and the renormalization group, Commun. Math. Phys. 210 (2000) 249-273, 216 (2001) 215-241; 
hep-th/9912092, hep-th/0003188; Insertion and elimination: the doubly infinite algebra of Feynman graphs, Ann. Inst. Henri Poincaré 3 (2002) 411-433; hep-th/0201157.

[DP] C. De Concini, C. Procesi, Wonderful models of subspace arrangements, Selecta Math. 1:3 (1995) 459-494.

[DF] M. Dütsch, K. Fredenhagen, Causal perturbation theory in terms of retarded products, and a proof of the action Ward identity, Rev. Math. Phys. 16:10 (2004) 1291-1348.

[EG] H. Epstein, V. Glaser, The role of locality in perturbation theory, Ann. Inst. H. Poincaré, A19:3 (1973) 211-295.

[EGS] H. Epstein, V. Glaser, R. Stora. General properties of the n-point functions in local quantum field theory, in: Structural Analysis of Collision Amplitudes, Les Houches 1975, R. Balian and D. Iagolnitzer eds, NorthHolland, Amsterdam; J. Bros, H. Epstein, V. Glaser, R. Stora, Quelques aspects globaux des problèmes d'Edge-of-the-Wedge, Nice 1973, in: Hyperfunctions and Theoretical Physics, Lecture Notes in Mathematics, Vol. 449, Berlin 1975, pp. 185-218.

[EGP] K. Ebrahimi-Fard, J.M. Gracia-Bondia, F. Patras, A Lie algebra approach to renormalization, Commun. Math. Phys. 276 (2007) 519549; hep-th/0609035.

[F] L. D. Faddeev and S. L. Shatashvili. Algebraic and Hamiltonian methods in the theory of nonabelian anomalies. Teoret. Mat. Fiz. 60:2 (1984) 206217 [English translation: Theoret. and Math. Phys. 60:2 (1984) 770-778]; L. D. Faddeev. Operator anomaly for the Gauss law. Phys. Lett. B145 (1984) 81-84.

[FHS] S. Falk, R. Häußling, F. Scheck, Renormalization in quantum field theory: an improved rigorous method, J. Phys. A: Math. Theor. 43 (2010) 035401 (15p); arXiv:0901.2252 [hep-th].

[FGG] S. Ferrara, R. Gatto, A.F. Grillo, Logarithmic scaling and spontaneous breaking, Phys.Lett. 42B (1972) 264-266.

[FG-B] H. Figeroa, J.M. Gracia-Bondia, Combinatorial Hopf algebras in quantum field theory, Rev. Math. Phys. 17 (2005) 881-; hep-th/0408145.

[FJL] D.Z. Freedman, K. Johnson, J.I. Latorre, Differential regularization and renormalization: a new method of calculation in quantum field theory, Nucl. Phys. B371 (1992) 353-314.

[FM] W. Fulton, R. MacPherson, A compactification of configuration space, Ann. Math. 139 (1994) 183-225. 
[GS] I.M. Gelfand, G.E. Shilov, Generalized Functions I, Academic Press, New York 1968 (original Russian edition: Moscow 1958).

[GPY] S. Giombi, S. Prakash, X. Yin, A note on CFT correlators in three dimensions, arXiv:1104.4317v3 [hep-th].

[G-B] J.M. Gracia-Bondia, Improved Epstein-Glaser renormalization in coordinate space I. Euclidean framework, Math. Phys. Anal. Geom. 6 (2003) 55-88; hep-th/0202023.

[G-BL] J.M. Gracia-Bondia, S. Lazzarini, Improved Epstein-Glaser renormalization in coordinate space II. Lorentz invariant framework, J. Math. Phys. 44 (2003) 3863-3875; hep-th/0212156.

[G-BVF] J.M. Gracia Bondía, J.C. Várilly, H. Figueroa, Elements of Noncommutative Geometry, Birkhäuser, 2000.

[HL] P.E. Haagensen, J.I. Latorre, Differential renormalization of massive quantum field theoris, Phys. Lett. B283 (1992) 293-297; hep-ph/9203207; A comprehensive coordinate space renormalization of quantum electrodynamics, Annals Phys. 221 (1993) 77-105; hep-ph/9206252.

[Hep] K. Hepp, Proof of the Bogoliubov-Parasiuk theorem on renormalization, Comm. Math. Phys. 2:4 (1966) 301-326; K. Hepp, La Théorie de la Renormalisation, Lecture Notes in Physics 2, Springer Verlag, Berlin 1969.

[H07] S. Hollands, The operator product expansion for perturbative quantum field theory in curved spacetime, Commun. Math. Phys. 273 (2007) 1-36; gr-qc/0605072.

[H08] S. Hollands, Renormalized quantum Yang-Mills fields in curved spacetime, Rev. Math. Phys. 20 (2008) 1033-1172; arXiv:0705.3340 [gr-qc].

[HW] S. Hollands, R.M. Wald, Existence of local covariant time ordered products of quantum fields in curved spacetime, Commun. Math. Phys. 231 (2002) 309-345; gr-qc/0111108.

[HW08] S. Hollands, R.M. Wald, Quantum field theory in curved spacetime the operator product expansion and dark energy, Gen. Rel. Grav. 40 (2008) 2051-2059; arXiv:0805.3419 [gr-qc].

[H] L. Hörmander, The Analysis of Linear Partial Differential Operators, I. Distribution Theory and Fourier Analysis, 2d edition, Springer, Berlin 1990 (see, in particular, Sect. 3.2 and Chapter VIII).

[K] K.J. Keller, Euclidean Epstein-Glaser renormalization, J. Math. Phys. 50 (2009) 103503; arXiv:0902.4789 [math-ph]. 
[K10] K.J. Keller, Dimensional regularization in position space and a forest formula for regularized Epstein-Glaser renormalization, arXiv:1006.2148 [math-ph].

[Kr] D. Kreimer, Knots and Feynman Diagrams, Cambridge Lecture Notes in Physics 13, Cambridge Univ. Press, Cambridge 2000.

[KTV] A.N. Kuznetsov, F.V. Tkachov, V.V. Vlasov, Techniques of distributions in perturbative quantum field theory I, hep-th/9612037.

[L] [10] Y. M. Lam, Equivalence theorem on Bogolyubov-Parasiuk-HeppZimmermann renormalized Lagrangian field theories, Phys. Rev. D7 (1973) 2943.

[LZ] J.H. Lowenstein, W. Zimmermann, On the formulation of theories with zero-mass propagators, Nuclear Phys. B86 (1975) 77-103.

[MZ] J. Maldacena, A. Zhiboedov, Constraining conformal field theories with higher spin symmetry, arXiv:1112.1016 [hep-th].

[Ni] N.M. Nikolov, Anomalies in quantum field theory and cohomologies in configuration spaces, arXiv:0903.0187 [math-ph]; Talk on anomaly in quantum field theory and cohomologies of configuration spaces, arXiv:0907.3735 [hep-th].

[N] N.M. Nikolov, Renormalization theory of Feynman amplitudes on configuration space, arXiv:0907.3734 [hep-th].

[NST] N.M. Nikolov, R. Stora, I. Todorov, Configuration space renormalization of massless QFT as an extension problem for associate homogeneous distributions, Bures-sur-Yvette preprint IHES/P/11/07 and work in progress.

[Pr] D. Prange, Causal perturbation theory and differential renormalization, J. Phys. A32 (1999) 2225-2238; hep-th/9710225.

[R] J. Rosner, Sixth order contribution to $Z_{3}$ in finite quantum electrodynamics, Phys. Rev. Letters 17:23 (1966) 1190-1192; Higher order contributions to the divergent part of $Z_{3}$ in a model quantum electrodynamics, Ann. of Physics 44 (1967) 11-34.

[Sc] L. Schwartz, Théorie des distributions I, II, Hermann, Paris 1950-51.

[Sp] E.R. Speer, On the structure of anlytic renormalization, Commun. Math. Phys. 23 (1971) 23-36; ibid. 25 (1972) 336.

[St] O. Steinmann, Perturbative Expansion in Axiomatic Field Theory, Lecture Notes in Physics, Springer 1971. 
[Step] B.M. Stepanov, Abstraktnaia teoriia R-operatsii, Izv. AN SSSR, ser. mat. 27 (1963) 819; On the construction of S-matrix in accordance with perturbation theory, Izv. AN SSSR, ser. mat. 29 (1965) 1037-1054 (transl. Amer. Math. Soc. (2) 91 (1969)).

[S82/93] R. Stora, Differential algebras in Lagrangean field theory, ETHZ Zürich Lectures, January-February 1993; G. Popineau, R. Stora, A pedagogical remark on the main theorem of perturbative renormalization theory, unpublished preprint (1982).

[S06] R. Stora, Causalité et groupes de renormalisation perturbatifs, in: T. Boudjedaa, A. Makhlouf (Eds.), Théorie Quantique des Champs Méthode et Applications, Ecole de Physique Théorique de Jijel, Algérie, 2006, Collection Travaux en cours, vol. 68, Editions Hermann 2007/2008.

[SP] E.C.G. Stueckelberg, A. Petermann, La normalization des constantes dans la théorie des quanta, Helv. Phys. Acta 26 (1953) 499-520.

[Stu] J. Lacki, G. Wanders, H. Ruegg (Eds.) Stueckelberg, an unconventional figure in twentieth century physics. Birkhäuser Verlag AG, 2009.

[U] N.I. Usyukina, Algorithm for calculating massless Feynman diagrams, Theor. Math. Phys. 79 (1989) 385-391 (transl from Teor. Mat. Fiz. 79 (1989) 63-71).

[Z] O.I. Zavyalov, Renormalized quantum field theory, Kluwer, Dordrecht 1990 (Russian original: Nauka, Moscow 1979).

[Zi] W. Zimmermann, Convergence of Bogoliubov's method of renormalization in momentum space, Commun. Math. Phys. 15 (1969) 208-234; Local operator products and renormalization in quantum field theory. In: S. Deser et al. (Eds.), Lectures on Elementary Particles and Quantum Field Theory, Proceedings of the 1970 Brandeis Summer Institute in Theoretical Physics, MIT Press, Cambridge, MA, pp. 399-589. 